\title{
Nobel Lecture: The fractional quantum Hall effect*
}

\author{
Horst L. Stormer \\ Department of Physics and Department of Applied Physics, Columbia University, New York, New York 10023 \\ and Bell Labs, Lucent Technologies, Murray Hill, New Jersey 07974
}

[S0034-6861(99)00704-7]

\section{INTRODUCTION}

The fractional quantum Hall effect is a very counterintuitive physical phenomenon. It implies that many electrons, acting in concert, can create new particles having a charge smaller than the charge of any individual electron. This is not the way things are supposed to be. A collection of objects may assemble to form a bigger object, or the parts may remain their size, but they don't create anything smaller. If the new particles were doubly charged, it wouldn't be so paradoxicalelectrons could "just stick together" and form pairs. But fractional charges are very bizarre indeed. Not only are they smaller than the charge of any constituent electron, but they are exactly $1 / 3$ or $1 / 5$ or $1 / 7$ etc. of an electronic charge, depending on the conditions under which they have been prepared. And yet we know with certainty that none of these electrons has split up into pieces.

Fractional charge is the most puzzling of the observations, but there are others. Quantum numbers-usually integers or half-integers - turn out to be also fractional, such as $2 / 5,4 / 9$, and $11 / 7$, or even 5/23. Moreover, bits of magnetic field can get attached to each electron, creating yet other objects. Such composite particles have properties very different from those of the electrons. They sometimes seem to be oblivious to huge magnetic fields and move in straight lines, although any bare electron would orbit on a very tight circle. Their mass is unrelated to the mass of the original electron but arises solely from interactions with their neighbors. More so, the attached magnetic field changes drastically the characteristics of the particles, from fermions to bosons and back to fermions, depending on the field strength. And finally, some of these composites are conjectured to coalesce and form pairs, vaguely similar to the formation of electron pairs in superconductivity. This would provide yet another astounding new state with weird properties.

All of these strange phenomena occur in twodimensional electron systems at low temperatures exposed to a high magnetic field-only electrons and a magnetic field. The electrons reside within a solid, at the interface between two slightly different semiconductors. This is presently the smoothest plane we can fabricate to restrict the electrons' motion to two dimensions. Quantum mechanics does the rest.

\footnotetext{
*The 1998 Nobel Prize in Physics was shared by Robert B. Laughlin, Horst L. Stormer, and Daniel C. Tsui. This lecture is the text of Professor Stormer's address on the occasion of the award.
}

Most of the experiments are very simple. Given a high magnetic field, typically from a commercial superconductive magnet, and given a temperature close to absolute zero, typically $1 / 100$ to $1 / 10$ of a degree Kelvin from a commercially available helium refrigerator, only a battery, a resistor, and a voltmeter are required. In reality one employs somewhat more sophisticated instrumentation to increase the data accumulation rate.

The samples are made from ultra-pure semiconductor materials. They are the essential ingredient for the experiments. Before diving into the mysterious caverns of two-dimensional many-particle physics, one needs to get an appreciation for the sophisticated technologies that make the journey possible.

\section{TWO-DIMENSIONAL ELECTRON SYSTEMS}

In a three-dimensional world, the creation of a twodimensional system usually requires a surface of an object or an interface between two substances and a force to keep things there. A game of billiards - on the surface of a table and held down by gravity-is a commonly cited model system. Electrons can be confined to the surface of liquid helium or to the surface of some insulator. They can be kept there by an electric field, which pushes them against a highly impenetrable barrier. The most successful method to create two-dimensional electron systems (2DESs) is to confine them within a solid to the interface between a semiconductor and an insulator or to the interface between two different semiconductors. The first is the so-called silicon MOSFET (metaloxide-semiconductor field-effect transistor), in which the 2DES is confined to the interface between silicon and silicon oxide; see Fig. 1(a).

In a silicon MOSFET, electrons reside at the silicon side of the interface, pushed against the highly impenetrable, insulating silicon oxide glass by an electric field from a metal electrode atop the glass. The ability to vary the electron concentration in the silicon-and hence the electrical resistance_-via the electrode (called the gate) makes this structure an ideal transistor. Silicon MOSFETs are the workhorse of today's $\sim \$ 140$ billion silicon industry - providing the central ingredient for everything from the $\mathrm{PC}$ to the digital watch.

In a MOSFET, electrons can move along the plane of the interface but are bound to it in the perpendicular direction. In fact, due to quantum mechanics, they cannot move in this direction at all. The electric field from the electrode pushes the carriers so strongly against the glass and they become so strongly entrapped in this direction that only a set of discrete states are quantum 
a)

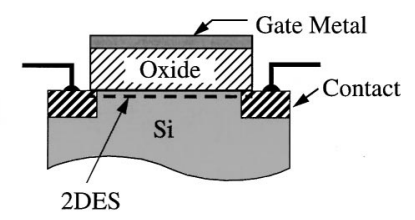

b)
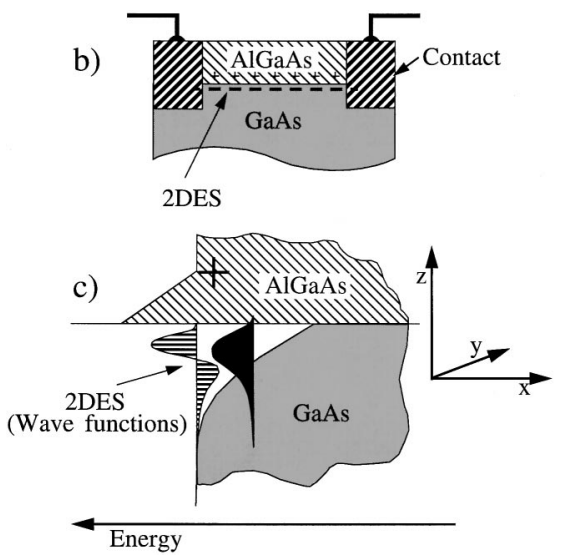

FIG. 1. Schematic drawings of a silicon metal-oxidesemiconductor field-effect transistor (MOSFET). (a) The twodimensional electron system (2DES) resides at the interface between silicon and silicon oxide. Electrons are held against the oxide by the electric field from the gate metal; (b) Schematic drawings of a modulation-doped gallium arsenide/ aluminum gallium arsenide (GaAs/AlGaAs) heterojunction. The 2DES resides at the interface between GaAs and AlGaAs. Electrons are held against the AlGaAs by the electric field from the charged silicon dopants $(+)$ in the AlGaAs; (c) Energetic condition in the modulation-doped structure (very similar to the condition in the MOSFET). Energy increases to the left. Electrons are trapped in the triangularshaped quantum well at the interface. They assume discrete energy states in the $z$ direction (black and horizontally striped). At low temperatures and low electron concentration only the lowest (black) electron state is occupied. The electrons are totally confined in the $z$ direction but can move in the $x-y$ plane.

mechanically allowed in this dimension [see Fig. 1(c)]. At low temperatures, much lower than the energetic spacing between these orbits, and at sufficiently low density, all electrons reside in the lowest of these states. Their behavior in this $z$ direction is rigidly confined. On the other hand, they are free to move in the $x-y$ plane. The silicon MOSFET represents an almost ideal implementation of the concept of a 2DES, and much of the physics of 2DESs has relied on it.

As good and versatile as they are, such MOSFETs have their limitations. Residing at the interface between a crystalline semiconductor and an insulating, random glass, electrons are often scattered by the roughness of the plane or by impurities that can penetrate the glass layer. Electron scattering is undesirable. It ejects electrons in a random fashion out of their trajectories, obscuring the observation of their "clean" behavior, governed solely by their mutual interactions and interactions with a magnetic field. Of course, electrons are also scattered by vibrations of the atoms, so-called phonons. Cooling the samples to temperatures near ab- solute zero reduces such vibrations to a level at which they become negligible as compared to scattering from any residual impurities.

Electrons, bound to the interface between two different crystalline semiconductors, should make for an even "better" 2DES than the one in a silicon MOSFET. Modulation-doped gallium-arsenide/aluminum-galliumarsenide (GaAs/AlGaAs) heterostructures have provided such a superior system for research and for some high-performance applications.

\section{MODULATION DOPING}

Pure semiconductors do not conduct electricity at low temperatures. There are no free electrons that can move about the crystal. All of them have been consumed by the bonds that hold the solid together. To conduct electricity, semiconductors require the addition of a small number of impurities, known as doping. Doping entails somewhat of a physical "catch 22": without doping there are no free electrons, but doping introduces impurities, which strongly scatter the newly introduced free carriers. In a three-dimensional semiconductor, this dilemma can practically not be circumnavigated. In two dimensions, however, there is a way. One can separate the mobile electrons from their parent impurities by confining them to different, neighboring planes. Such layers need to be in close proximity to each other for the impurities to transfer their electrons, but sufficiently far apart to prevent such electrons from scattering off the charged core of their bare parent impurities, which they leave behind. Molecular beam epitaxy (MBE) provides the tools for such an undertaking.

MBE is basically a high-vacuum evaporation technique which allows one to evaporate high-quality, thin layers of semiconductors onto each other. Invented in the late 1960s by Al Cho at Bell Laboratories, it forms the basis of a large industry, manufacturing highperformance photonic and electronic devices, with an emphasis on communications. One standard materials combination used in MBE crystal growth in GaAs and GaAlAs. These are two semiconductors with practically identical atom-to-atom spacing (lattice constant), but they differ slightly in the energies of their free electrons (electron affinity). Electrons have a slight "preference" for GaAs over AlGaAs-about $300 \mathrm{meV}$ in a typical sandwich. An almost identical lattice constant guarantees a virtually defect-free, stress-free, and hence highquality interface. The difference in electron affinity allows one to keep electrons at bay from their highly scattering parent impurities.

In its most common implementation, the 2DES in an MBE-grown GaAs/AlGaAs sandwich resides at the GaAs side of a single interface with AlGaAs; see Fig. 1(b). A several- $\mu$ m-thick GaAs layer is grown onto a 1/2-mm-thick GaAs substrate. The substrate provides a template for the arriving atoms as well as mechanical support for the final structure. The GaAs layer is then covered by a $\sim 0.5-\mu \mathrm{m}$-thick layer of AlGaAs. During the high-quality, extremely clean, atomic-layer-by- 
atomic-layer growth process, silicon impurities are introduced into the AlGaAs material at a distance of about $0.1 \mu \mathrm{m}$ from the interface. Each silicon impurity has one more outer-shell electron than the gallium atom, which it replaces in the solid. It easily loses this additional electron, which wanders around the solid as a conduction electron. Seeking the energetically lowest state, the electron ventures over the energetic cliff and falls "down" into the GaAs material, only $0.1 \mu \mathrm{m}$ away. In the highly pure GaAs layer such conduction electrons can move practically unimpeded by their parent silicon impurities, which remain in the AlGaAs layer, on the other side of the barrier. With modulation doping you "can have your cake and eat it."

The attraction from all those positively charged (loss of one electron) stationary silicon ions pulls the mobile electrons against the AlGaAs barrier of the interface [see Fig. 1(c)]. The conditions are completely analogous to the conditions in a Si MOSFET, in which the metal gate pulls the electrons against the silicon oxide barrier of its interface. The same quantization of the $z$ motion of the carriers arises and the carriers become quantum mechanically bound to the interface, but remain mobile within the $x-y$ plane. The advantage that a modulationdoped GaAs/AlGaAs heterostructure provides over a $\mathrm{Si}$ MOSFET originates from its atomically smooth interface between two crystalline semiconductors of very high purity. Transistors from such modulation-doped material (so-called HEMT transistors) represent today's lowest-noise, highest-frequency transistors and are extensively used in mobile telephony. Amazingly, much of the bizarre physics to be described below would occur in a transistor, not unlike those in many mobile phones, if cooled to low temperatures and placed in a high magnetic field.

Electron mobility is a common measure for the ease with which electrons move through a material. At low temperatures, where the scattering by phonons is negligible, mobilities in today's GaAs/AlGaAs heterostructures exceed those in Si MOSFETs by almost a factor of 1000 ! Such modulation-doped specimens represent presently the best implementation of the concept of a twodimensional metal, almost free of detrimental scattering from the host (see Fig. 2). This fact is best expressed as a mean free path of an electron before it scatters. It is $\sim 1 / 5 \mathrm{~mm}$, meaning that a conduction electron passes by one million atoms of the semiconductor without scattering.

Modulation doping was invented and implemented in 1977 by four researchers at Bell Laboratories. Figure 3 shows a photograph taken around that time, in which they congregate around an early MBE machine. MBE technology has advanced immensely since these early days, and MBE machines have grown in size and complexity. Figure 4 shows a photograph of today's highmobility MBE system at Bell Labs and the researchers who employ it to fabricate the world's most exquisite modulation-doped specimens.

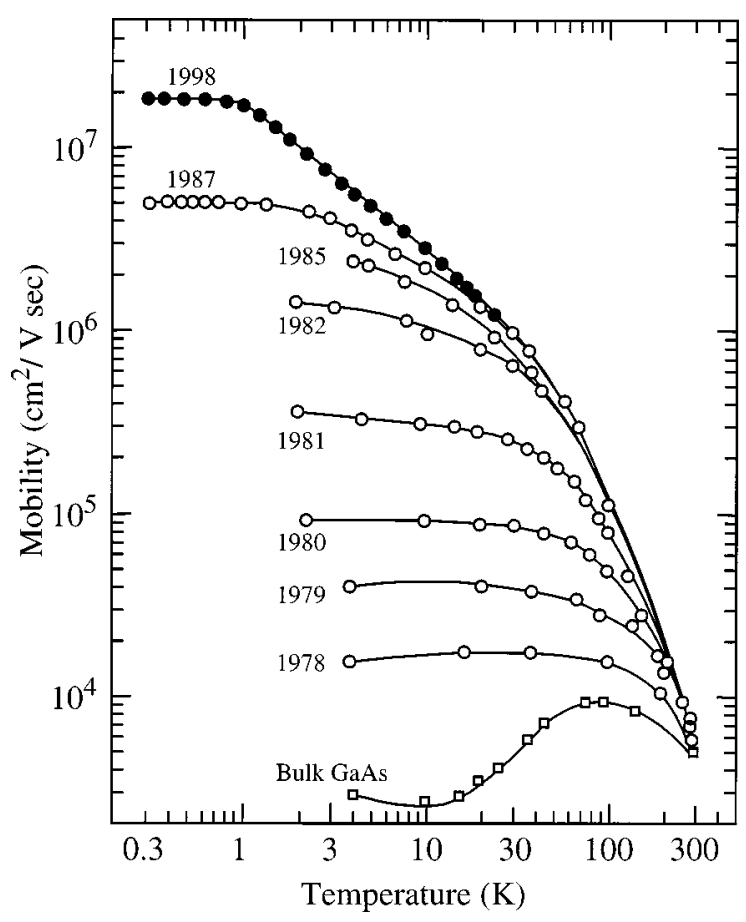

FIG. 2. Progress made over the years in the mobility $(\mu)$ of electrons in two-dimensional electron systems in modulationdoped GaAs/AlGaAs as a function of temperature. At high temperature $\mu$ is limited by scattering with phonons of the solid. At the lowest temperatures $\mu$ is limited by impurities and defects in the material. "Bulk GaAs" represents a characteristic bulk sample. Since the inception of modulation doping $\mu$ has risen by more than a factor of 1000 . A mobility of 2 $\times 10^{7} \mathrm{~cm}^{2} /$ Vsec corresponds roughly to $1 / 5 \mathrm{~mm}$ (!) ballistic flight of the electrons through the semiconductor before a collision takes place.

\section{THE HALL EFFECT}

The Hall effect was discovered in 1879 in a sheet of gold leaf by Edwin Hall, a graduate student at Johns Hopkins University in Baltimore. Running a current $I$ through such a thin metal sheet, he measured two char-

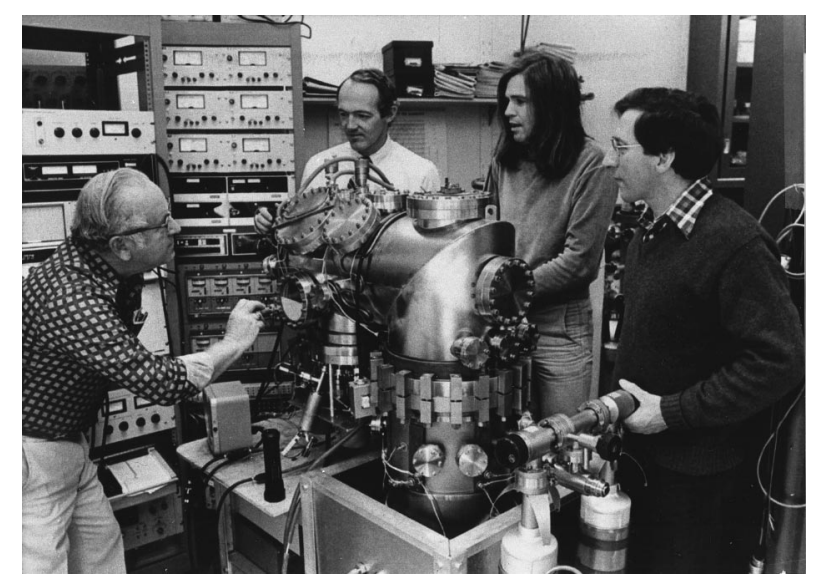

FIG. 3. The inventors of the modulation-doping process congregating in 1978 around an early molecular beam machine at Bell Labs. From left: Willy Wiegmann, Art Gossard, Horst Stormer, and Ray Dingle. 


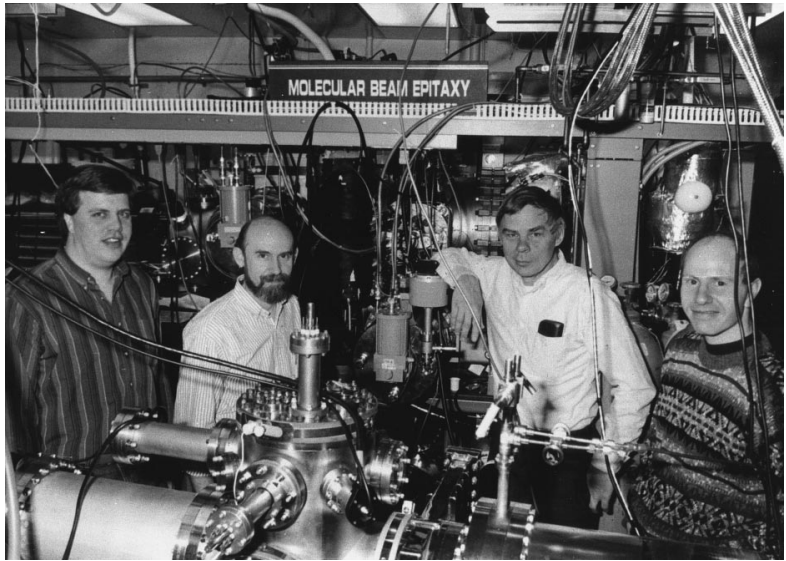

FIG. 4. Today's Bell Labs ultra-high-purity molecular beam epitaxy equipment with Loren Pfeiffer (center right) and Ken West (center left), who are synthesizing the world's highestmobility material. They are joined by Kirk Baldwin (left), who has worked with me for almost 20 years, and Amir Yacoby (right), a postdoc who worked on one-dimensional wires.

acteristic voltages (see Fig. 5). The first, $V$, was the voltage along the current path, which, when divided by the current, represented the electrical resistance $R$ of the material. The second, $V_{H}$, was the voltage across the current path, which was expected to be zero since the current ran perpendicular to it. This was indeed Hall's observation until he applied a magnetic field $B$ vertical to the metal sheet. It gave rise to a nonzero voltage $V_{H}$ across the current path. From his different experiments, Hall deduced that $V_{H}$ was proportional to the current $I$ and proportional to the magnetic field $B$. Hence, denoting $V_{H} / I$ as an electrical resistance $R_{H}$ yielded $R_{H} \propto B$. Ever since this effect has been known as the Hall effect.

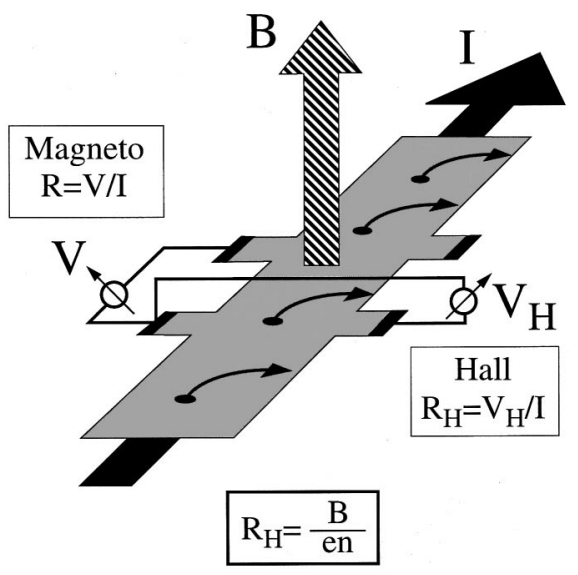

FIG. 5. Geometry for measurement of the magnetoresistance $R$ and the Hall resistance $R_{H}$ as a function of the current $I$ and magnetic field $B$. $V$ represents the longitudinal voltage, which is dropping along the current path, and $V_{H}$ the Hall voltage, which is dropping perpendicular to the current path. The electron density per $\mathrm{cm}^{2}$ is denoted as $n$ and the charge of the electron as $e$. The black dots represent electrons that are forced toward one side of the bar following the Lorentz force from the magnetic field.
The associated voltage $V_{H}$ is the Hall voltage, which, when divided by the current $I$, becomes the Hall resistance $R_{H}$.

The origin of the Hall effect is classical electrodynamics. The presence of the magnetic field exerts a sideward force (Lorentz force) onto the electrons, which, on average, had been moving in the direction of the current. They are pushed toward one side of the specimen (depending on the direction of the magnetic field), giving rise to a charge accumulation on one side as compared with the other. This accumulation of charge ultimately results in the appearance of a voltage across the current path. Obviously, the higher the field, the bigger the push, the bigger $R_{H}$. But also, the lower the density of electrons, the higher $R_{H}$. This sounds initially counterintuitive, but is rather simple, too. To generate the same current, fewer electrons need to travel faster. Faster electrons experience a stronger Lorenz force and create a bigger $V_{H}$ and, hence, a bigger $R_{H}$.

In its final form $R_{H}=B /(n e)$, where $n$ is the electron density per $\mathrm{cm}^{2}$ (unit area) in the sample, which is equal to the electron density $N$ per $\mathrm{cm}^{3}$ (unit volume) times the thickness of the specimen, and $e$ is the elementary charge of an electron. Notice that no other electron parameter, such as its mass, nor any of the material parameters are entering - only the electron density. Most remarkably, $R_{H}$ does not depend on the shape of the specimen. In fact, even a set of holes drilled into the specimen would not alter the result. A perforated metal sheet shows the same Hall resistance as a perfect sheet, as long as all electrical contacts remain mutually connected. Due to its independence from all intrinsic and extrinsic parameters, the Hall effect has become a standard tool for the determination of the density of free electrons in electrical conductors. In particular, the electron density of semiconductors, which can vary widely, depending on preparation, is measured via the Hall effect.

In 1879 Edwin Hall discovered that in a normal conductor the resistance $R_{H}$ depends linearly on the strength $B$ of the magnetic field (see Fig. 6). In 1980 Klaus von Klitzing discovered that, for the case of twodimensional electron systems, the dependence is very different.

\section{THE INTEGRAL QUANTUM HALL EFFECT}

Perform a Hall experiment at the low temperature of liquid $\mathrm{He}(\sim 4 \mathrm{~K})$ in a very high magnetic field $(\sim 10 \mathrm{~T})$ on the two-dimensional electron system of a $\mathrm{Si}$ MOSFET and you will find a stepwise dependence of the Hall resistance on magnetic field, rather than Edwin Hall's linear relationship (see Fig. 7). Yet more surprisingly, the value of $R_{H}$ at the position of the plateaus of the steps is quantized to a few parts per billion (!) to $R_{H}=h /\left(i e^{2}\right)$, where $i$ is an integer and $h$ is Planck's constant $\left(R_{H} \approx 25.812 \ldots k \Omega\right.$ for $\left.i=1\right)$. In $1990, h / e^{2}$, the quantum of resistance, as measured reproducibly to eight significant digits via this integral quantum Hall effect (IQHE), became the world's new resistance stan- 


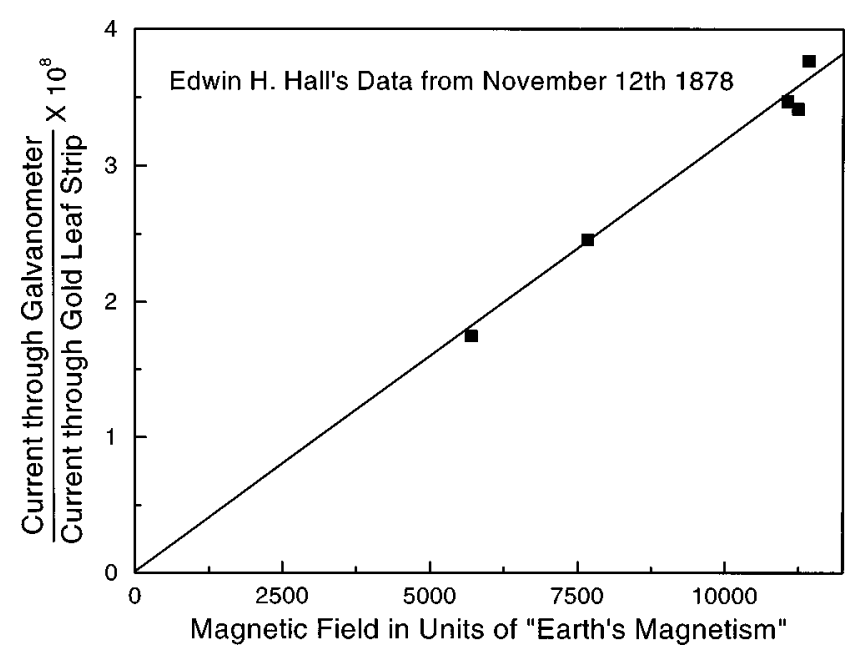

FIG. 6. Edwin Hall's Hall data of 1878 as plotted from a table in his publication. The vertical axis is proportional to the Hall voltage $V_{H}$ of Fig. 5 and the horizontal axis is proportional to the magnetic field of Fig. 5. A linear relationship between $V_{H}$ and $B$ and hence between $R_{H}$ and $B$ is apparent. Since the days of Edwin Hall, this strictly linear relationship has been confirmed by many, much more precise experiments.

dard. Concomitant with the quantization of $R_{H}$, the magnetoresistance $R$ drops to vanishingly small values. This is another hallmark of the IQHE and both are directly related.

Why are two-dimensional systems (2DESs) so different? And what is the origin of the steps and minima? Classically, electrons in a high magnetic field are forced onto circular orbits, following the Lorentz force. Quantum mechanically, there exists only a discrete set of allowed orbits at a discrete set of energies. The situation is
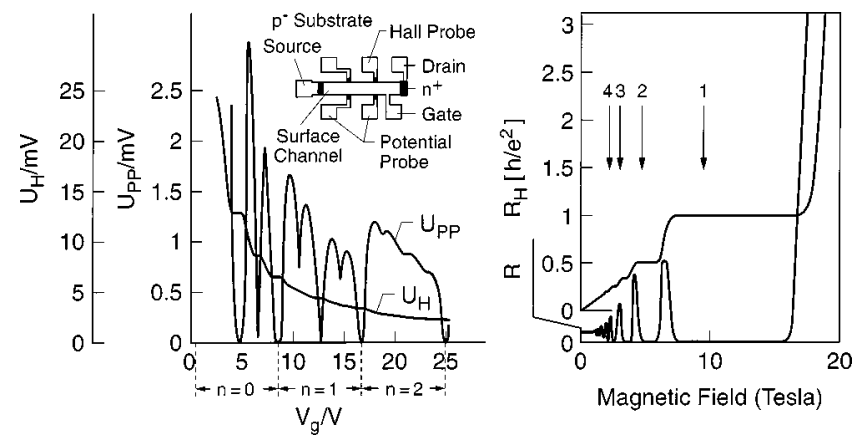

FIG. 7. The integral quantum Hall effect. Left panel: original data of the discovery of the integral quantum Hall effect (IQHE) by Klaus von Klitzing in 1980 in the two-dimensional electron system of a silicon MOSFET transistor. Instead of a smooth curve, he observed plateaus in the Hall voltage $\left(U_{H}\right)$ and found concomitant deep minima in the magnetoresistance $\left(U_{P P}\right)$. The horizontal axis represents gate voltage $\left(V_{G}\right)$, which varies the carrier density $n$. The right panel shows equivalent data taken on a two-dimensional electron system in GaAs/AlGaAs. Since these data are plotted vs magnetic field, they can directly be compared to Edwin Hall's data of Fig. 6. Rather than the linear dependence of the Hall resistance on magnetic field of Fig. 6, these data show wide plateaus in $R_{H}$ and in addition deep minima in $R$. not unlike the discrete set of orbits that arise in an atom. Energetically, these so-called Landau levels represent an equally spaced ladder of states having energies, $E_{i}=(i$ $\left.-\frac{1}{2}\right) h e B /(2 \pi m) \quad(i=1,2,3, \ldots)$, proportional to the magnetic field $B$. Here $m$ is the electron mass and $h$ is Planck's constant. (Throughout this lecture we are neglecting the effects due to the electron spin. It simplifies the discussion without much loss of generality.) Electrons can only reside at these energies, but not in the large energy gaps in between. The existence of the gaps is crucial for the occurrence of the IQHE. Here 2DESs differ decisively from electrons in three dimensions. Motion in the third dimension, along the magnetic field, can add any amount of energy to the energy of the Landau levels. Therefore, in three dimensions, the energy gaps are filled up and hence eliminated, preventing the quantum Hall effect from occurring. In 2DESs, in addition to the existence of energy gaps, the number of electrons fitting into each Landau level is exactly quantized. It reflects the number $d$ of orbits that can be packed per Landau level into each $\mathrm{cm}^{2}$ of the specimen. This turns out to be $d=e B / h$. Notice that this capacity per Landau level, also called its degeneracy, apart from natural constants, depends only on the magnetic field $B$. None of the materials parameters enters in any way. It is therefore a universal measure, independent of the material employed.

Let the sample have a fixed $2 \mathrm{D}$ electron density $n$. At low temperatures, where all electrons try to fall into the energetically lowest available states, and in a sufficiently high magnetic field, all electrons fit into the lowest Landau level, filling it only partially. As the field is lowered, the capacity of the Landau levels shrink according to $d$ $=e B / h$. At $B_{1}=n h / e$ the lowest Landau level is exactly full. Any further reduction of the field requires the first electron to leave the lowest Landau level and jump across the energy gap to the next higher Landau level at an energy cost of he $B_{1} /(2 \pi m)$. Reducing the field to $B_{2}=(n h / e) / 2=B_{1} / 2$ fills two Landau levels, and the first electron has to move to the third level, etc. This creates a sequence of fields $B_{i}=(n h / e) / i$, at which all electrons fill up an exact number of Landau levels, keeping all higher Landau levels exactly empty. At these special points on the magnetic-field axis, the magnetoresistance $R$ drops momentarily and the Hall resistance $R_{H}$ assumes a set of very special values. Using $R_{H}=B /$ (ne) from the classical Hall resistance and inserting the values of the sequence of distinctive fields $B_{i}$ into the equation results in a quantized Hall resistance of $R_{H}$ $=h /\left(i e^{2}\right), i=1,2,3 \ldots$. While this is the desired result, it does not account for the true hallmarks of the IQHE, which are wide plateaus in $R_{H}$ and broad minima in $R$.

According to the above derivation, $R_{H}$ would take on its quantized value only at very precise positions $B_{i}$ of magnetic field. This would be a poor basis for a standard, since the precision to which $R_{H}$ assumes one of the quantized values would depend on the precision to which one could determine $B$. In reality, in the IQHE, the Hall resistance $R_{H}$ assumes the quantized values over extended regions of $B$ around $B_{i}$. 


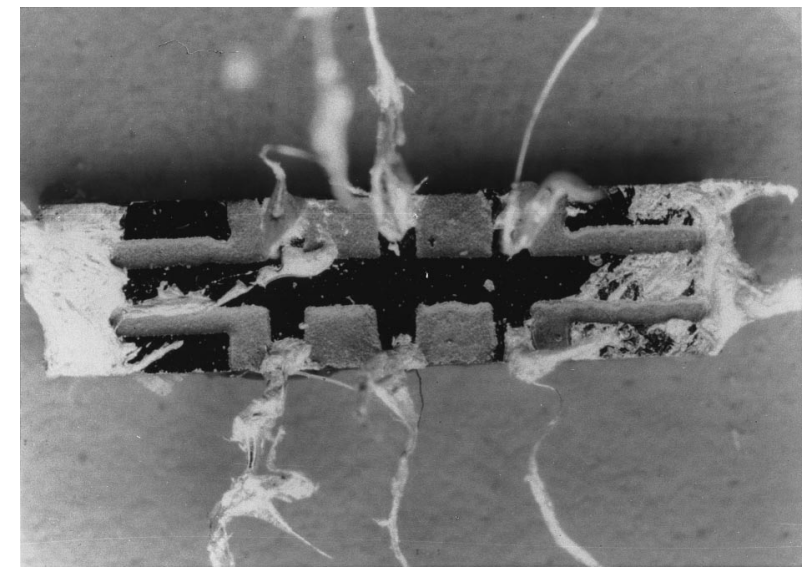

FIG. 8. Photograph of a GaAs/AlGaAs sample. The size is about $6 \times 1.5 \mathrm{~mm}$. Black area (in reality mirrorlike but reflecting the black camera) is the original surface above the 2DES. Gray areas have been scratched away to confine the current path to the center of the sample. White areas are indium blotches used to make contact with the 2DES. Gold wires are attached. Specimens like this one, prepared with little attention to exact dimension or to tidiness, show quantization of the Hall resistance to an accuracy of about 10 parts in a billion. The specimen shown is the sample in which the fractional quantum Hall effect (FQHE) was discovered in 1981.

The origin for plateau formation and broad minima lies in electron localization. In spite of the extreme care with which the 2DES is prepared, there remain some energetic valleys and hillocks along the interface, be they due to residual defects, steps, or impurities. Each Landau level is a reproduction of this uneven landscape. As a Landau level is being filled with electrons, some of the electrons get trapped (localized) and isolated. They no longer participate in the electrical conduction through the specimen, and these patches of localized electrons become inert and act like a set of holes cut out from the $2 \mathrm{D}$ sheet. As in a perforated metal sheet, such isolated patches do not affect the measurements of the density of mobile carriers in the flat part of the landscape, which are circumnavigating the hills and valleys. As long as filling and emptying of a Landau level fills or empties only the localized states at the energetic fringes, while keeping the Landau level in the extended flat regions full to capacity, the sample's Hall resistance $R_{H}$ and magnetoresistance $R$ remain steady. Since, in the conducting regions, the Landau level is full, the Hall resistance remains fixed to its quantized value. Localized electrons provide a reservoir of carriers that keep the Landau levels in the energetically flat part of the sample exactly filled for finite stretches of magnetic field, giving rise to finite stretches of quantized Hall resistance and vanishing resistance in the IQHE.

The precision of quantization does not depend on the shape and size of the specimen, nor on the particular care taken to define its contact regions. (Figure 8 shows a particularly egregious example.) In a quirk of nature, the existence and precision of the IQHE plateaus requires the existence of imperfections in the sample. Without such dirt there would be no IQHE. Instead, even in a 2DES, one would revert to Edwin Hall's straight line.

In an ingenious thought experiment, Bob Laughlin was able to deduce the existence and precision of the IQHE from a set of very simple experimental ingredients (see his contribution to this volume). In his approach, the value of $R_{H}=h /\left(i e^{2}\right)=(h / e) /(i e)$ emerges as a ratio of the magnetic flux quantum $\phi_{0}=h / e$ and the electronic charge $e$, together with the number of occupied Landau levels $i$. Magnetic flux quanta are the elementary units in which a magnetic field interacts with a system of electrons. (The magnetic field itself is not quantized. This is different from charge, which usually comes in chunks of $e$. However, for the purposes of this lecture, which deals with magnetic fields in the presence of electrons, one may think of it as being quantized.) Being the ratio of $\phi_{0}$ to $e$, one can regard $R_{H}$ as being a very precise measure of the electron charge when expressed as $e=\phi_{0} /\left(i R_{H}\right)$. From this purview, Klaus von Klitzing's experiment has provided a highly accurate electrometer to determine the charge of the currentcarrying particle in a 2DES.

\section{THE FRACTIONAL QUANTUM HALL EFFECT}

\section{Discovery}

In the beginning of October, 1981, Dan Tsui and I, both working at Bell Labs, had taken a specimen of a new sample made from modulation-doped GaAs/ AlGaAs material to the Francis Bitter Magnet Lab at MIT in Cambridge. The sample had been grown by Art Gossard, also of Bell Labs, and his assistant Willie Wiegmann. Having gained increasing experience with modulation doping over the course of a couple of years, they had, for the first time, been able to fabricate a lowelectron-density sample $\left(n=1.23 \times 10^{11} \mathrm{~cm}^{-2}\right)$ with an exceedingly high mobility of $\mu=90000 \mathrm{~cm}^{2} / \mathrm{V} \mathrm{sec}$. Figure 8 is actually a photograph of this specimen. Given the high magnetic fields available at the magnet lab, we foresaw being able to venture into the so-called extreme quantum limit, where the lowest Landau level is only partially occupied with electrons. The goal was to investigate this regime for signs of the so-called Wigner solid, an electron crystal in two dimensions. The formation of such a regular array of electrons had been predicted theoretically, but remained unobserved.

On October 7, a Hall measurement on this specimen at the temperature of liquid $\mathrm{He}(4.2 \mathrm{~K})$ produced the data at the top of Fig. 9. The largely linear relationship between Hall resistance $R_{H}$ and magnetic field $B$ is evident. Deviations at low field indicate the emergence of the IQHE. Knowledge of the electron density, as well as the values of the resistance steps $\left[R_{H}=h /\left(i e^{2}\right), i\right.$ $=1,2,3 \ldots]$, clearly identify these features as the IQHE. With the last $(i=1)$ step occurring at $B \approx 5 \mathrm{~T}(\sim 7 \mathrm{~cm}$ on the $\mathrm{mm}$ paper), for all fields beyond this point the electrons had to reside in the lowest Landau level, filling it to only a fraction $\nu$ of its capacity. As the sample was cooled to $1.5 \mathrm{~K}$, the IQHE features firmed up, develop- 


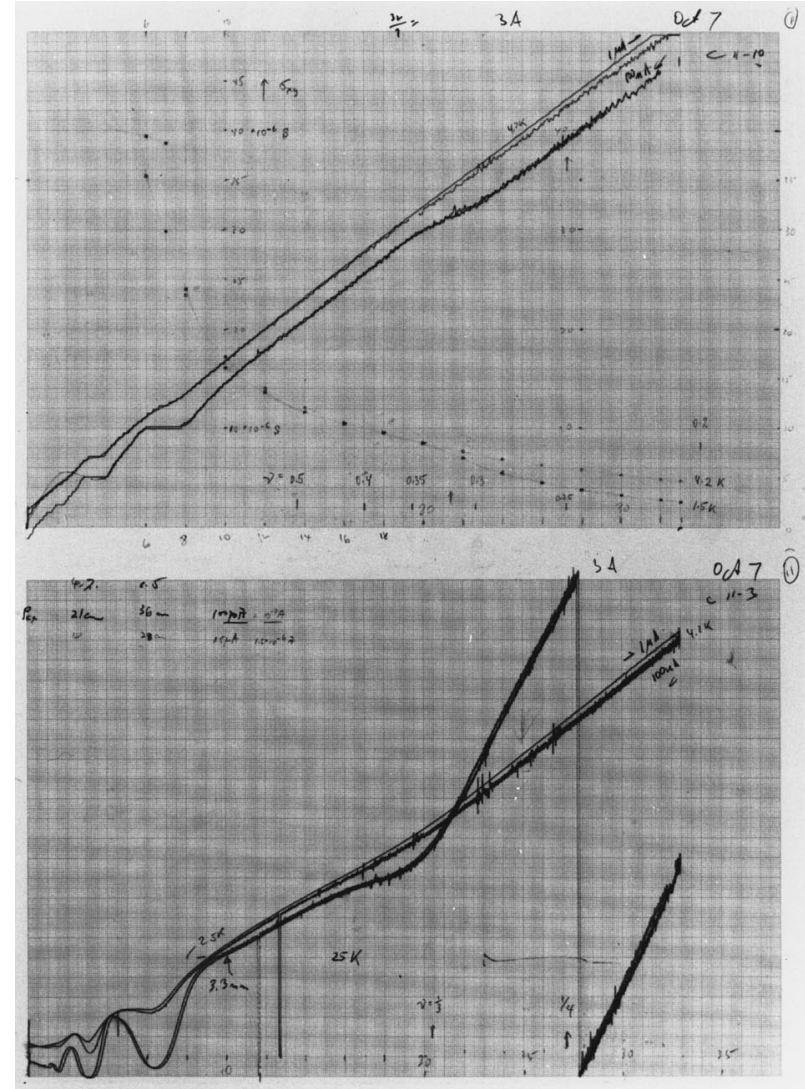

FIG. 9. Data of October 7, 1981, on the specimen \# 6-19-81(3) (see Fig. 8) on millimeter paper. The top panel shows the Hall resistance $R_{H}$ at temperatures $4.2 \mathrm{~K}$ and $1.5 \mathrm{~K}$ vs magnetic field $B$. The bottom panel shows the magneto resistance $R$ vs $B$ at similar temperatures. $1 \mathrm{~T}$ is equivalent to $\sim 1.5 \mathrm{~cm}$. Features at $\sim 3 \mathrm{~cm}$ and $\sim 7 \mathrm{~cm}$ are due to the IQHE. Weaker features at $\sim 21 \mathrm{~cm}$ are due to the FQHE. The scribbles in the top panel are from reuse of the millimeter paper for data reduction from other traces.

ing the familiar, flat plateaus (see top Fig. 9). A remarkable feature occurred at $B=15 \mathrm{~T}$ : the Hall trace started to deviate from the originally straight line, showing a behavior not unlike that observed in the IQHE at the higher temperature of $4.2 \mathrm{~K}$. This feature was totally unexpected. Beyond the emergence of a plateau in $R_{H}$, the magnetoresistance $R$ seemed to exhibit a concomitant minimum (see bottom Fig. 9).

The IQHE, arising from exact filling of Landau levels, could not have been at work, since above $B \approx 5 \mathrm{~T}$ the lowest level was only partially occupied. Furthermore, the Hall resistance in the vicinity of this change in slope far exceeded the largest possible of IQHE resistances of $R_{H}=h / e^{2} \approx 25 \mathrm{k} \Omega$. Lightheartedly, Dan Tsui enclosed the distance between $B=0$ and the position of the last IQHE $(\sim 7 \mathrm{~cm})$ between two fingers of one hand and measured the position of the new feature in this unit. $\mathrm{He}$ determined it to be three and exclaimed, "quarks!" Although obviously joking, with finely honed intuition, he had hit on the very essence of the data.

Following Laughlin's gedanken experiment and accepting quantization of the Hall resistance to measure the charge of the particle, a plateau three times as high

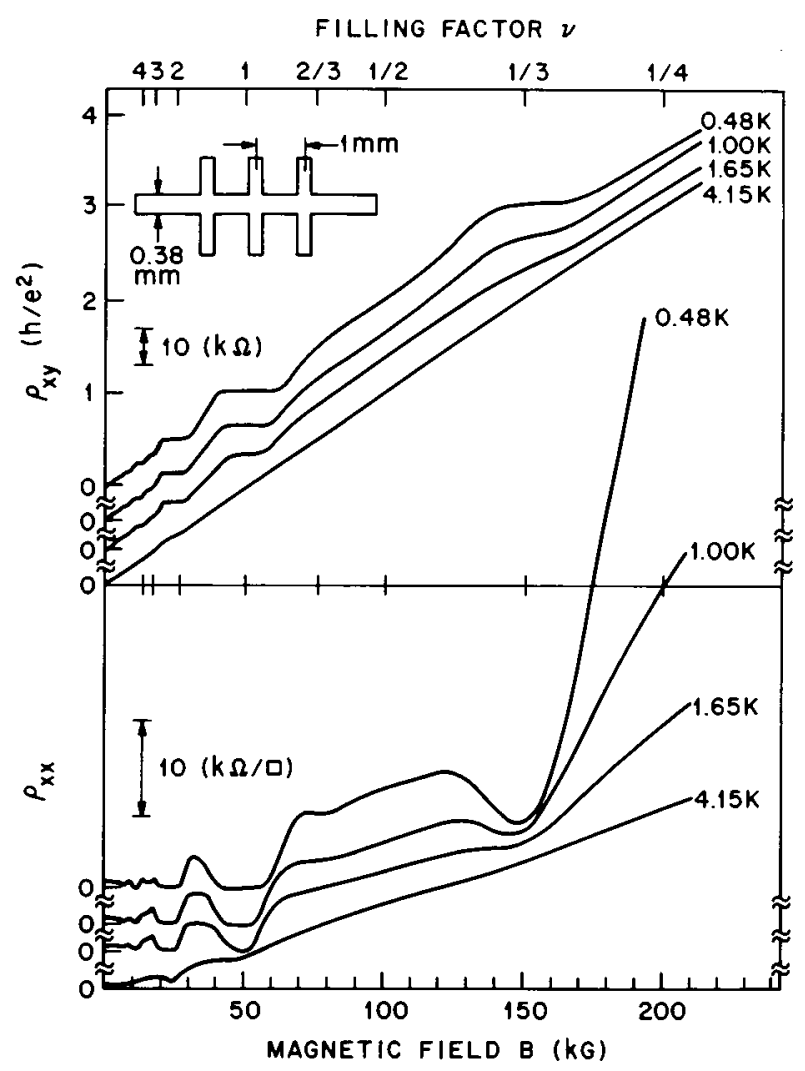

FIG. 10. First publication on the FQHE. Hall resistance data (here $\rho_{x y}$ ) and magnetoresistance data (here $\rho_{x x}$ ) are from the same specimen as in Fig. 9. The filling factor $\nu$ of the Landau level is indicated on the top. The features at $\nu=1,2,3, \ldots$ are due to the IQHE. The features at $\nu=1 / 3$ are due to the FQHE. Sample dimensions and sample temperatures are indicated.

as the last IQHE plateau meant the appearance of a charge $q=\phi_{0} /\left(3 h / e^{2}\right)=e / 3$. Obviously, our lowtemperature, low-energy experiment (milli-eV, not Millions-eV) could not have generated anything even remotely related to quarks (subnuclear particles endowed with $1 / 3$ charge), but, as it turned out, the implication of some kind of fractionally charged particle was dead right. At the time, we did not know what we had discovered. The paper on the findings (see Fig. 10), published in March 1982 in Physical Review Letters with Tsui, Stormer, and Gossard as authors, speculated on its being a signature of a Wigner solid or equivalent, but the paper also remarked on a fractional charge. No photographs exist from the days of October 1981. However, Fig. 11 is a record of our first ultra-low-temperature ultra-high-magnetic-field run at the Francis Bitter National Magnet Lab at MIT.

\section{Origin}

The IQHE can be understood solely on the basis of the quantized motion of individual 2D electrons in the presence of a magnetic field and random fluctuations of the interface potential which creates localized states. The existence of all fellow electrons enters only in the simplest of ways-as a filler of empty states of the Lan- 


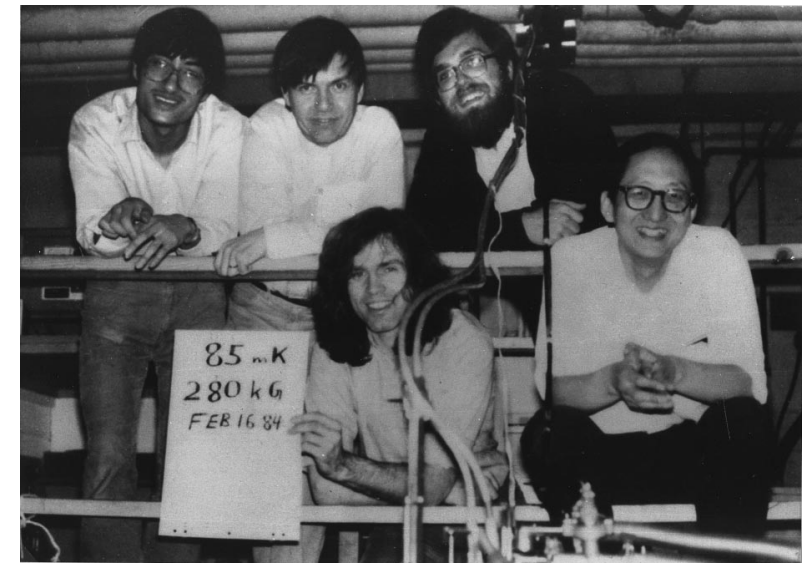

FIG. 11. First successful operation of our dilution refrigerator in high magnetic field. The sign reads: $85 \mathrm{mK}, 280 \mathrm{kG}$, Feb. 16. 84. The proud operators are, clockwise from upper left, Albert Chang, Peter Berglund (who was largely responsible for the design and implementation of the instrument), Greg Boebinger, Dan Tsui, and Horst Stormer.

dau levels. The electrostatic interaction (so-called Coulomb interaction) between the like-charged carriers is irrelevant to the understanding of the IQHE. It is therefore called a single-particle effect.

The FQHE (fractional quantum Hall effect), on the other hand, can no longer be understood on the basis of the behavior of individual electrons in a magnetic field. The existence of an energy gap-so crucial for the exact quantization in the IQHE-is expected also to be essential for the occurrence of the FQHE. However, all magnetic-field-induced energy gaps have been exhausted by the IQHE and have emerged as integral quantization of the Hall resistance to $R_{H}=h /\left(i e^{2}\right), i$ $=1,2,3 \ldots$. Other energy gaps, at fractional filling of a Landau level, must be of a different origin.

The origin of the FQHE is interaction between electrons. It is therefore termed a many-particle effect or an electron correlation effect, since the charged electrons are avoiding each other by correlating their relative motion in an intricate manner. In the IQHE, electrons have no freedom to avoid one another. Occurring at exact integral Landau-level filling, electrons are already "close packed" with no option for further avoidance. At fractional filling this is different. There is much "space" in a Landau level. Electrons have the freedom to avoid each other in the energetically most advantageous fashion. The electron solid we had been searching for, in which electrons reside at fixed positions of maximum mutual distance, would have represented a static pattern that minimizes electron interaction. In the FQHE, the electrons assume an even more favorable state, unforeseen by theory, by conducting an elaborate, mutual, quantum-mechanical dance.

Many-particle effects are extraordinarily challenging to address theoretically. In most situations they cause only a small adjustment of the behavior of the electrons and can be taken into account in an approximate manner. Often such a treatment is quite adequate, but on occasion many-particle interactions become the essence of a physical effect. Superconductivity and superfluidity are of such intricate origin. To account for their occurrence one had to devise novel, sophisticated theoretical means. The emergence of the FQHE requires such a new kind of thinking.

Bob Laughlin had the correct theoretical insight and invented an elegant wave function which described the quantum-mechanical behavior of all those electrons in the 1/3 FQHE (as well as all other 1/q FQHE states) in a very succinct equation of some 15 letters. It represents a triumph of many-particle theory. $\mathrm{He}$ also provided a reason for the existence of an energy gap and a derivation of this most mysterious charge of $e / 3$ (see his contribution to this issue). In the following sections I will attempt to give the reader an impression of the simple beauty of the physical concepts in the regime of the FQHE. Rather than addressing the expert, to whom several excellent monographs are available (see bibliography), my presentation aims at the scientifically knowledgeable layperson who attempts to develop a sense for the origin of a phenomenon as strange as the FQHE. The discussion follows a nonhistorical path. It draws from the concept of the formation of composite particles between electrons and the magnetic field. From the vista of this model, the serene beauty of electron correlation in 2D Landau levels manifests itself most clearly.

\section{Of electrons and flux quanta}

In a classical model, 2D electrons behave like charged billiard balls on a table [Fig. 12(a)]. They are distinguishable by virtue of their different history and they can be tracked individually. Quantum mechanically, electrons are smeared out over the table. They are inherently indistinguishable and one can only cite a probability of finding an electron-any electron-at any particular location. In a perfect $2 \mathrm{D}$ system, this probability is absolutely uniform over the whole plane. The electrons behave like a featureless liquid [Fig. 12(b)]. That is not to say that the motions of the electrons are not correlated. These like-charged carriers strongly avoid each other, as shown in Fig. 12(c) in a classical representation. They also do this in the quantum-mechanical liquid of Fig. 12(b). It affects the probability of finding one electron here having detected another electron there (e.g., close by), but one cannot represent it in a graph as simple as Fig. 12(c).

It was an important conceptual step to realize that an impinging magnetic field $B$ could be viewed as creating tiny whirlpools, so-called vortices, in this lake of charge-one for each flux quantum $\phi_{0}=h / e$ of the magnetic field [Fig. 12(d)]. The notion of a whirlpool is quite appropriate, since such vortices have indeed a quantummechanical "swirl"- a phase twist- to them. Inside the vortex, electronic charge is displaced, dropping to zero in the center and recovering to the average surrounding charge density at the edge of the vortex. The extent of a vortex is roughly the size of the area which contains one quantum of magnetic flux $\left(\operatorname{area} \times B=\phi_{0}\right)$. Therefore each vortex can be thought of as carrying with it one flux 
a)

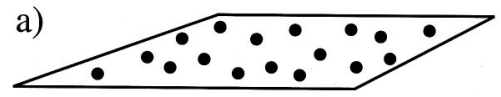

2D electrons

classical

\section{b)}
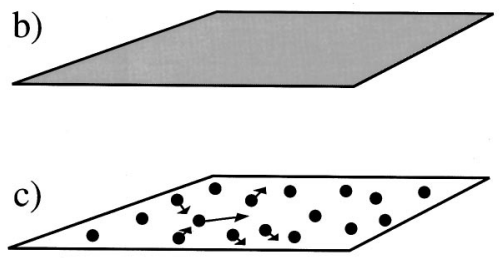

electron-electron correlation classical

d)

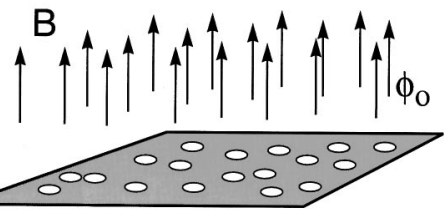

$2 \mathrm{D}$ electrons in a magnetic field (schematically)

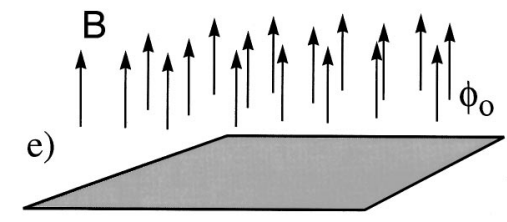

$2 \mathrm{D}$ electrons in a magnetic field (really)

FIG. 12. Schematic drawing of 2DES in various approximations. Black dots represent electrons. White holes represent vortices. Arrows represent magnetic flux quanta $\phi_{0}$ of the magnetic field $B$.

quantum. Of course, just as the electrons are spread out uniformly over the plane, so are the vortices. As required by quantum mechanics, the probability of finding an electron-as well as a vortex-remains totally uniform [Fig. 12(e)]. However, the picture of electrons and vortices provides an intuitive way of looking at electronelectron correlation in the presence of a magnetic field.

Electrons and vortices are opposite objects, one representing a package of charge, the other the absence of charge. Correlation of their mutual positions can prove energetically very beneficial. Placing vortices directly onto electrons is particularly advantageous since the trough of the whirlpool, which represents the displacement of all fellow electrons, keeps their charges at bay and reduces mutual repulsion. This intuitive image requires some mental flexibility. Each electron is at the center of a vortex and at the same time is part of the pool of electrons generating vortices surrounding all those other electrons. Who says many-particle physics is easy?

Each electron always needs to be surrounded by one vortex. In the language of electrons and vortices, it is the system's way of satisfying the Pauli exclusion principle for electrons, which, in this situation, requires that no two electrons can be in the same position. The whirlpool provides the required place of respite. At complete filling of the lowest Landau level, where the number of electrons equals the number of flux quanta, the arrangement of electrons and vortices is totally controlled by the Pauli principle-one vortex per electron, no choices (see Fig. 13). This is the condition of the $i=1 \mathrm{IQHE}$. It

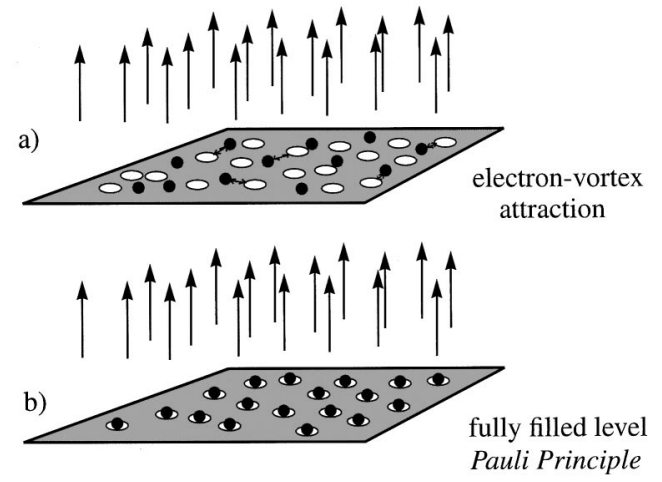

FIG. 13. Schematic drawing of electron vortex attraction of a 2DES in the presence of a magnetic field: (a) In the fully filled Landau level $\nu=1$, there are as many vortices as there are electrons, and the Pauli exclusion principle forces the vortices onto the electrons (b). (The spin of the electron is neglected throughout.)

can easily be extended to more Landau levels and also to include both electron-spin directions and hence to $i$ $=2,3,4 \ldots$. The IQHE is driven by the Pauli exclusion principle for electrons. It is another way of expressing that the existence of other electrons enters the IQHE only in the simplest of ways-as a filler of empty states. When the number of vortices deviates from the number of electrons, then there are choices.

At magnetic fields higher than the $i=1 \mathrm{IQHE}$, the stronger magnetic field provides more flux quanta and hence there are more vortices than there are electrons. The Pauli principle is readily satisfied by placing one vortex onto each electron [Fig. 14(a)] - but there are more vortices available. The electron system can considerably reduce its electrostatic Coulomb energy by placing more vortices onto each electron [Fig. 14(b)]. More vortices on an electron generate a bigger surrounding whirlpool, pushing further away all fellow electrons, thereby reducing the repulsive energy. The so-

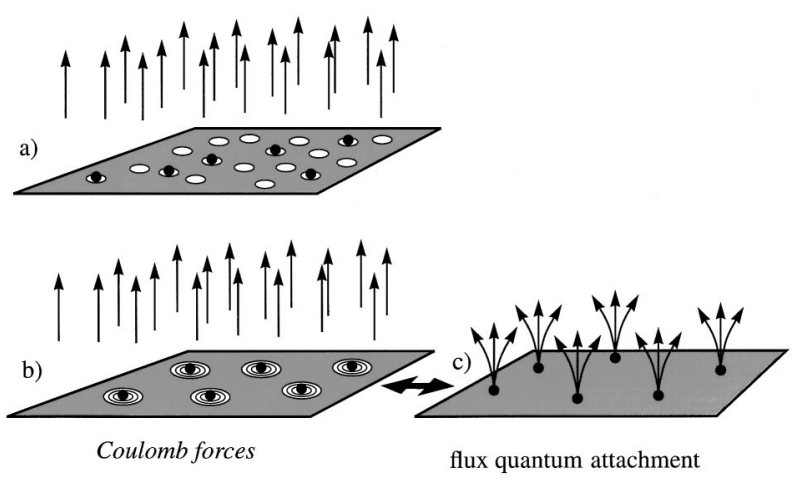

FIG. 14. Schematic drawing of electron vortex attraction at fractional Landau-level filling $\nu=1 / 3$. Now there are three times as many vortices as there are electrons. (a) The Pauli principle is satisfied by placing one vortex onto each electron. (b) Placing three vortices onto each electron reduces electronelectron (Coulomb) repulsion. Vortex attachment can be viewed as the attachment of magnetic flux quanta to the electrons, transforming them to composite particles (c). 
established relative motion of electrons is no longer driven by the Pauli exclusion principle but by reduction in Coulomb energy. This is the central principle underlying electron-electron correlation in 2DES in a magnetic field. Casting electron-electron correlation in terms of vortex attachment facilitates the comprehension of this intricate many-particle behavior. Regarding the vortices as little whirlpools ultimately remains a crutch for visualizing something that has no classical analog.

\section{Composite particles}

Vortices are the expression of flux quanta in the 2D electron system, and each vortex can be thought of as having been created by a flux quantum. Conceptually, it is advantageous to represent the vortices simply by their "generators," the flux quanta themselves. Then the placement of vortices onto electrons becomes equivalent to the attachment of magnetic flux quanta to the carriers [Fig. 14(b),(c)]. Electrons plus flux quanta can be viewed as new entities, which have come to be called composite particles, CPs. As these objects move through the liquid, the flux quanta act as an invisible shield against other electrons. Replacing the system of highly interacting electrons by a system of electrons with such a "guard ring"-compliments of the magnetic field-removes most of the electron-electron interaction from the problem and leads to composite particles which are almost void of mutual interactions. It is a minor miracle that such a transformation from a very complex manyparticle problem of well-known objects (electrons in a magnetic field) to a much simpler single-particle problem of rather complex objects (electrons plus flux quanta) exists and that it was discovered.

CPs act differently from bare electrons. All of the external magnetic field has been incorporated into the particles via flux quantum attachment to the electrons. Therefore, from the perspective of CPs, the magnetic field has disappeared and they no longer are subject to it. They inhabit an apparently field-free 2D plane. Yet more importantly, the attached flux quanta change the character of the particles from fermions to bosons and back to fermions.

\section{Fermions and bosons}

In physics one differentiates between two types of particles, bosons and fermions. Fermions, such as electrons or protons, have the property that all other such particles are excluded from being in the same quantummechanical state, e.g., in the same position. They are subject to the Pauli exclusion principle and fill sequentially one available state after the other. Bosons, such as photons or helium atoms, have no such restriction and even have a preference for being in the same state. They follow Bose-Einstein statistics. In a very casual way, the exclusion principle for fermions is the reason for the world's not collapsing (all identical fermions staying away from each other) and the second is the origin for phenomena such as lasing or superfluidity (all photons or helium atoms condensing into the same state), usually referred to as Bose condensation. Fermions have halfinteger spin while bosons have integer spin-spin being related to the "spinning" of the particle.

As the case of superfluidity in helium shows, fermions, the elements of atoms (electrons, protons, and neutrons), can be assembled to "make" bosons (helium atoms). In a casual way, superconductivity, too, can be seen as the assembly of pairs of fermions (electrons) into bosons (Cooper pairs) which Bose condense into a superconducting state. One cannot assemble bosons to make "quasi" fermions. In a very simplistic way, the reason for the difference is that half-integers can be added to make integers but integers cannot be added to make half-integers.

Systems of fermions and systems of bosons behave very differently under mutual exchange of the position of two of their constituent particles-it is said to change their statistics. Their wave function-the quantummechanical description of the behavior of all the particles-is multiplied by -1 in the case of fermions and by +1 in the case of bosons. It is one of the deeper mysteries of quantum mechanics and cannot be further illuminated here. In any case, one needs to accept Nature's teachings.

\section{Composite particle statistics}

Electrons are fermions. As one slowly moves two electrons in a 2D electron system around each other and exchanges them, the wave function undergoes the sign reversal expected from fermions. It is different for CPs (Fig. 15). The attached flux quanta need to be taken into account, and their presence changes the particles' statistics. As one slowly moves two CPs around each other and exchanges them, the electrons by themselves reverse the sign of the wave function, but each attached flux quantum creates an extra "twist," multiplying it by an extra -1. As a result, CPs can be either fermions or bosons, depending on the number of attached flux quanta. An electron plus an even number of flux quanta becomes a composite fermion (CF), since the wave function is multiplied by -1 an odd number of times, i.e., by -1 . An electron plus an odd number of flux quanta becomes a composite boson (CB), since the wave function is multiplied by -1 an even number of times, i.e., by +1 . This so-called transmutation of the particle statistics through flux quantum attachment is deeply rooted in the two-dimensionality of the system. It represents a deep connection between space and particle statistics.

Accepting that CPs incorporate the external magnetic field and show either boson or fermion behavior, the perplexing properties of $2 \mathrm{D}$ electron systems in a high magnetic field can readily be appreciated.

\section{1/3 Fractional quantum hall state}

At $1 / 3$ filling of the lowest Landau level $(\nu=1 / 3)$, the magnetic field contains three times as many flux quanta per unit area as there are electrons in the $2 \mathrm{D}$ system. 

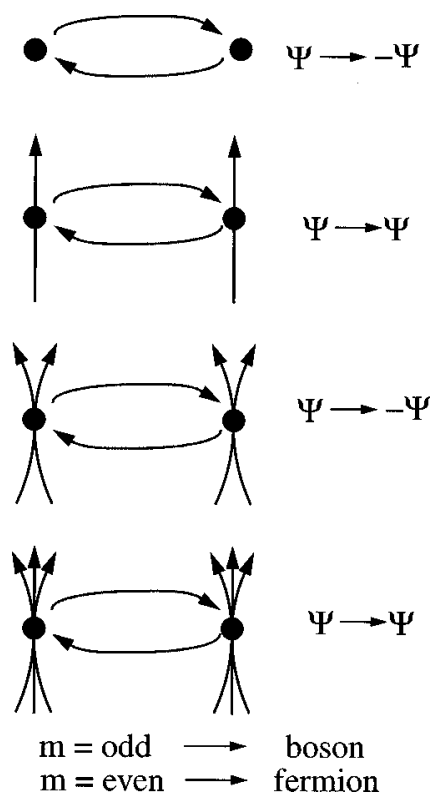

FIG. 15. Statistics of electrons and composite particles. Exchange of two particles affects the wave function $\Psi$, which describes the quantum-mechanical behavior of the system. For electrons, $\Psi$ is multiplied by -1 , identifying the particles as fermions. With the attachment of an odd number of flux quanta, $\Psi$ remains unchanged under exchange (multiplication by +1 ), identifying these particles as bosons. Attachment of an even number of flux quanta returns the particles to fermions. Here $m$ is the number of flux quanta.

Therefore the electron liquid contains three times as many vortices as there are carriers. To minimize electron-electron interaction, each electron accepts three vortices, which keeps fellow electrons optimally at bay. This is equivalent to the attachment of three magnetic flux quanta to each electron, which renders these objects CPs [Fig. 14(c)]. Since all the external magnetic field has been incorporated into the particles, they reside in an apparently magnetic-field-free region. Consisting of an electron plus an odd number of flux quanta, the resulting composites are composite bosons (CBs). Being bosons and residing in apparently zero magnetic field, these CBs Bose condense into a new ground state with an energy gap, characteristic for such Bose condensation. This is the sought-after energy gap required for quantization of the Hall resistance and vanishing resistance to arise. It has been measured by various experimental techniques, most directly by light scattering.

As the magnetic field deviates from exactly $\nu=1 / 3$ filling to higher fields, more vortices are being created (Fig. 16). They are not attached to any electrons, since this would disturb the symmetry of the condensed state. The amount of charge deficit in any of these vortices amounts to exactly $1 / 3$ of an electronic charge. These quasiholes (whirlpool in the electron lake) are effectively positive charges as compared to the negatively charged electrons. An analogous argument can be made for magnetic fields slightly below $\nu=1 / 3$ and the creation of quasielectrons of negative charge $e / 3$. Quasiparticles can move freely through the $2 \mathrm{D}$ plane and transport electrical current. They are the famous $1 / 3$ charged par-

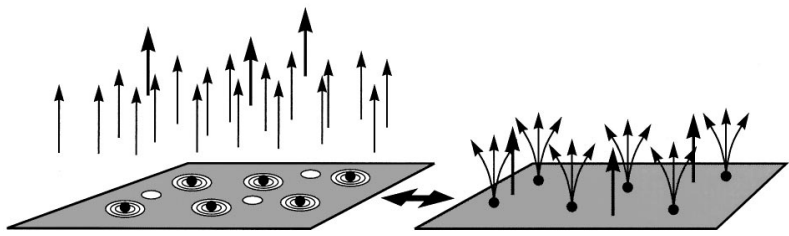

FIG. 16. Schematic representation of $1 / 3$ charged quasiparticles. At slightly higher $B$ fields than at $\nu=1 / 3$ additional vortices are created. They represent dimples in the electron lake. In the dimples exactly $1 / 3$ of an electron charge is missing. These are the fractionally charged quasiparticles of the FQHE.

ticles of the FQHE that have been observed by various experimental means, most recently by measurement of the amount of electrical noise that they generate. Plateau formation in the FQHE arises, in analogy to plateau formation in the IQHE from potential fluctuations and the resulting localization of carriers. In the case of the FQHE the carriers are not electrons, but, instead, the bizarre fractionally charged quasiparticles.

The FQHE at $\nu=1 / 5,1 / 7$, etc., with quasiparticles of charge $e / 5, e / 7$, etc., can be accounted for in total analogy to the $1 / 3 \mathrm{FQHE}$ by attaching 5,7 , etc. flux quanta to each electron. In fact, even states at $\nu=2 / 3,4 / 5,6 / 7$, etc. and $\nu=1+1 / 3,1+1 / 5$, etc. can be covered by this procedure, regarding, for example, the $\nu=2 / 3$ state as a full Landau level with $1 / 3$ missing electrons. In this way all fractions at Landau-level filling factor $\nu=i \pm 1 / q$ (often called the primary fractions) can be rationalized. But there are many others.

The state at $\nu=1 / 2$

At first sight, the $\nu=1 / 2$ state should be similar to the $1 / 3$ state, yet it turns out to be very different. At half filling of the lowest Landau level, the magnetic field contains two times as many flux quanta per unit area and hence creates two times as many vortices as there are carriers. In analogy to the $1 / 3$ state, each electron accepts now two vortices, which keeps the others at bay (Fig. 17). However, the attachment of an even number of magnetic flux quanta to each electron renders these objects composite fermions (CFs) and not composite bosons. This drastically changes their behavior as compared to the $1 / 3 \mathrm{FQHE}$ and its equivalents.

As at $\nu=1 / 3$ so also at $\nu=1 / 2$, the entire external magnetic field has been incorporated into the particles and they reside at apparently zero magnetic field. However,

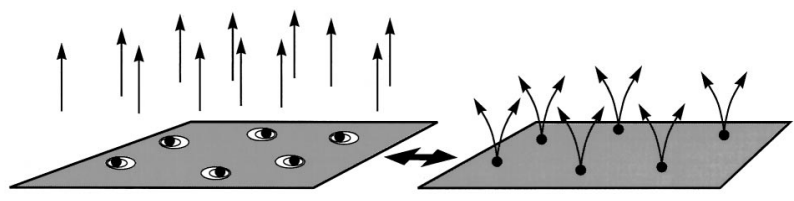

FIG. 17. Schematic representation of the state at Landau-level filling factor $\nu=1 / 2$. Two vortices are bound to each electron, equivalent to the attachment of two flux quanta. The slight offset of the second vortex is meant to represent the formation of tiny in-plane dipoles. 


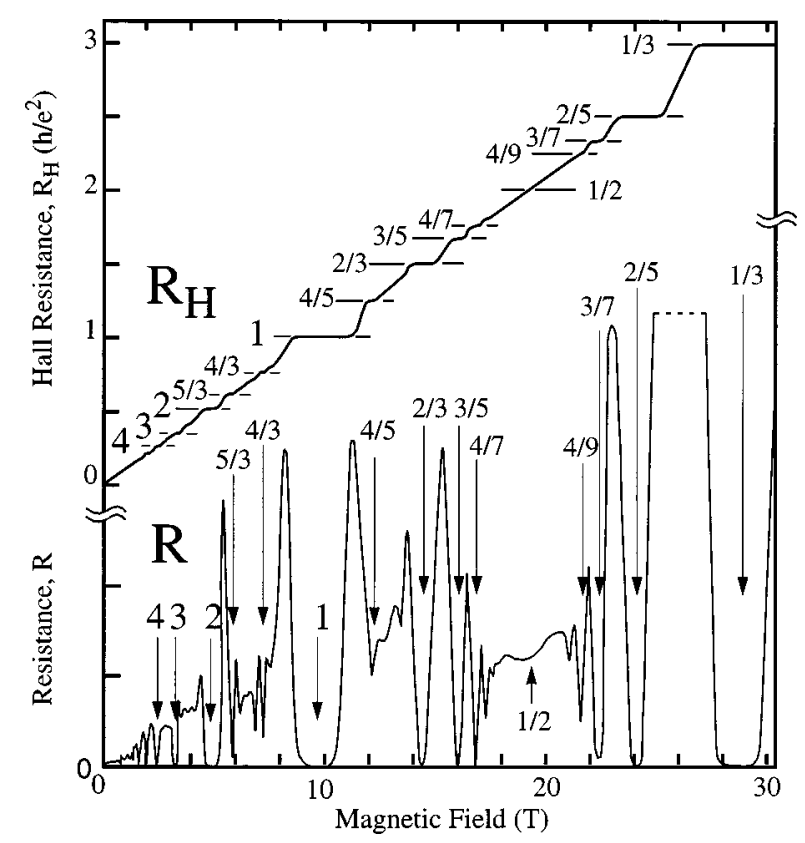

FIG. 18. The FQHE as it appears today in ultrahigh-mobility modulation-doped GaAs/AlGaAs 2DESs. Many fractions are visible. The most prominent sequence, $\nu=p /(2 p \pm 1)$, converges toward $\nu=1 / 2$ and is discussed in the text.

being fermions they are prevented from condensing into the lowest energy state. Instead, they fill up successively the sequence of lowest-lying energy states, until a maximum is reached and all CFs have been accommodated. The process is equivalent to the filling of states by electrons at $B=0$. Hence, from the point of view of CFs, the $\nu=1 / 2$ state appears equivalent to the case for electrons at $B=0$. In spite of the huge external magnetic field at half filling of the Landau level, CFs are moving in a similar fashion to electrons moving in zero field. This has been directly observed in experiment. Flux quantum attachment has transformed these earlier electrons and they are propagating along straight trajectories in a high magnetic field, where normal electrons would orbit on very tight circles. The mass of a $\mathrm{CF}$, usually considered to be a property of the particle, is unrelated to the mass of the underlying electron. Instead, the mass depends on the magnetic field and only on the magnetic field. In fact, it is a mass of purely many-particle origin, arising solely from interactions, rather than being a property of any individual particle. It is another one of these baffling implications of $e$-e interactions in high magnetic fields. The absence of condensation and the lack of an energy gap prevents the $\nu=1 / 2$ state from showing a quantized Hall resistance. Instead the Hall line is featureless, just as it is for electrons around $B=0$ (see Fig. 18).

The difference between $\nu=1 / 3$ and $\nu=1 / 2$ is striking. One is a Bose-condensed many-particle state showing a quantized Hall effect and giving rise to fractionally charged particles. The other is a Fermi sea, in spite of the existence of a huge external field, and its particles have a mass that arises from interactions. One flux quantum per electron makes all the difference.
There are many fascinating open questions associated with the $\nu=1 / 2$ state, such as: how does the mass vary with energy for CFs? and what is the microscopic structure of the particles? Also, how does the electron spin (which we were neglecting throughout this lecture) affect $\mathrm{CF}$ formation? A beautiful picture of composite fermions being tiny dipoles is emerging. While one of the vortices is placed directly on the electron (Pauli principle), the position of the second vortex is a bit displaced from exact center, rendering the object an electric dipole in the $2 \mathrm{D}$ plane. There is great promise for future discovery and future theoretical insight.

\section{All those other FQHE states}

Bose condensation of CBs consisting of electrons and an odd number of flux quanta rationalizes the appearance of the FQHE at the primary fractions around Landau-level filling factor $\nu=i \pm 1 / q$ with quantized Hall resistances $R_{H}=h /\left(\mathrm{v} e^{2}\right)$ and deep minima in the concomitant magnetoresistance $R$. However, a multitude of other FQHE states have been discovered over the years. Figure 18 shows one of the best of today's experimental traces on a specimen with a multimillion $\mathrm{cm}^{2} / \mathrm{V} \mathrm{sec} \mathrm{mo-}$ bility. What is the origin of these other states? The composite fermion model offers an extraordinarily lucid picture. We shall discuss it for the sequence of prominent fractions $2 / 5,3 / 7,4 / 9,5 / 11, \ldots$ and $2 / 3,3 / 5,4 / 7,5 / 9, \ldots$. (i.e., $\nu=p /(2 p \pm 1), p=2,3,4 \ldots$ ) around $\nu=1 / 2$.

At half filling the electron system has been transformed into CFs consisting of electrons which carry two magnetic flux quanta. All of the external magnetic field has been incorporated into the particles and they reside in an apparently field-free 2D plane. Since they are fermions, the system of CFs at $\nu=1 / 2$ resembles a system of electrons of the same density at $B=0$. What happens as the magnetic field deviates from $B=0$ ? For electrons their motion becomes quantized into electron-Landau orbits. They fill up their electron-Landau levels, encounter the energy gaps, and exhibit the well-known IQHE. CFs around $\nu=1 / 2$ follow the same route. As the magnetic field deviates from exactly $\nu=1 / 2$, the motion of CFs becomes quantized into CF-Landau orbits. They fill up their CF-Landau levels, encounter CFenergy gaps, and exhibit an IQHE. However, this is not an IQHE of electrons, but an IQHE of $C F$ s. This IQHE of CFs arises exactly at $\nu=p /(2 p \pm 1)$, which are the positions of the FQHE features. In fact, the oscillating features in the magnetoresistance $R$ of the FQHE around $\nu=1 / 2$ closely resemble the oscillating features in $R$ around $B=0$ and, once they have been shifted from $B=0$ to $\nu=1 / 2$, they coincide with their position. This is very remarkable in several ways.

CFs "survive" the additional (effective) magnetic field (away from $\nu=1 / 2$ ), and the orbits of these composite particles mimic the orbits of electrons in the equivalent magnetic field in the vicinity of $B=0$. The CFs remain "good" particles. In this way, a complex electron manyparticle problem at some rational fractional filling factor has been reduced to a single-particle problem at integer 
filling of CF-Landau levels in an effective magnetic field. Even the variation of the size of the energy gaps from one FQHE state to the next can be regarded as deriving from the ladder of Landau levels of CFs. More strikingly yet, excellent quantum-mechanical wave functions for these FQHE states can simply be derived from electron Landau levels. Therefore the FQHE of electrons can be regarded as the IQHE of CFs.

The CF model has been extraordinarily successful in conquering those other FQHE states. Even the 1/3 state can be viewed from the vantage point of this model. At $\nu=1 / 3$ the CFs emanating from $\nu=1 / 2$ have been quantized into CF-Landau levels and they are exactly filling the lowest of these levels. Hence the $\nu=1 / 3 \mathrm{FQHE}$ state is the equivalent of the $i=1 \mathrm{IQHE}$ of CFs which had formed at $\nu=1 / 2$. In analogy to the electron case, the flux quanta-one per $\mathrm{CF}$ - create vortices in the CF liquid which are forced onto the CFs to satisfy the Pauli principle for CFs. Each electron, holding two flux quanta from being a $\mathrm{CF}$ in the first place, acquires a third one, exactly as required to become the $\nu=1 / 3$ state. Bose condensation of CBs reappears as Landaulevel formation in CFs. In the FQHE regime they represent two different sides of the same coin.

With all these similarities, is the FQHE then the same as the IQHE? Certainly not. From one point of view the FQHE is the result of a complex behavior (manyparticle interactions) of simple particles (electrons) in the presence of a true external magnetic field. From another point of view it is the simple behavior (Landau quantization) of complex new multicomponent particles (composite fermions) in the presence of an effective magnetic field. This view of the FQHE has developed over the past decade or so. Its extreme simplicity is testament to the beauty of nature as much as it demonstrates the ability and intuition of dozens of theorists who have shaped it over the years. Whatever model one constructs for the FQHE, its origin is an elegant quantum-mechanical dance of electrons in a magnetic field.

CFs are "everywhere." All even-denominator fractions are candidates for $\mathrm{CF}$ formation. And all those $\mathrm{CF}$ states are capable of generating their own CF Landau levels, leading to a panoply of FQHE states. Such other states are already visible in Fig. 18. Yet more of such states appear in Fig. 19. FQHE states are emanating from $1 / 2,3 / 2,1 / 4,3 / 4,5 / 4$, and possibly from $3 / 8$ and $3 / 14$. There does not seem to be an end, although eventually the most fragile of states are destroyed by residual potential fluctuations, or by the formation of solids of electrons or CFs. Yet better 2DESs should remove the curtain from those sensitive fractions. Their mere detection and classification may appear somewhat unimaginative. However, so far, whenever we dug a bit deeper into the rich soil of the 2DES in the presence of a magnetic field we were rewarded with more surprises. One of them has already occurred.

\section{The peculiar state at $\nu=5 / 2$}

Electrons with two attached flux quanta are fermions. They fill up sequentially the lowest energy states and are

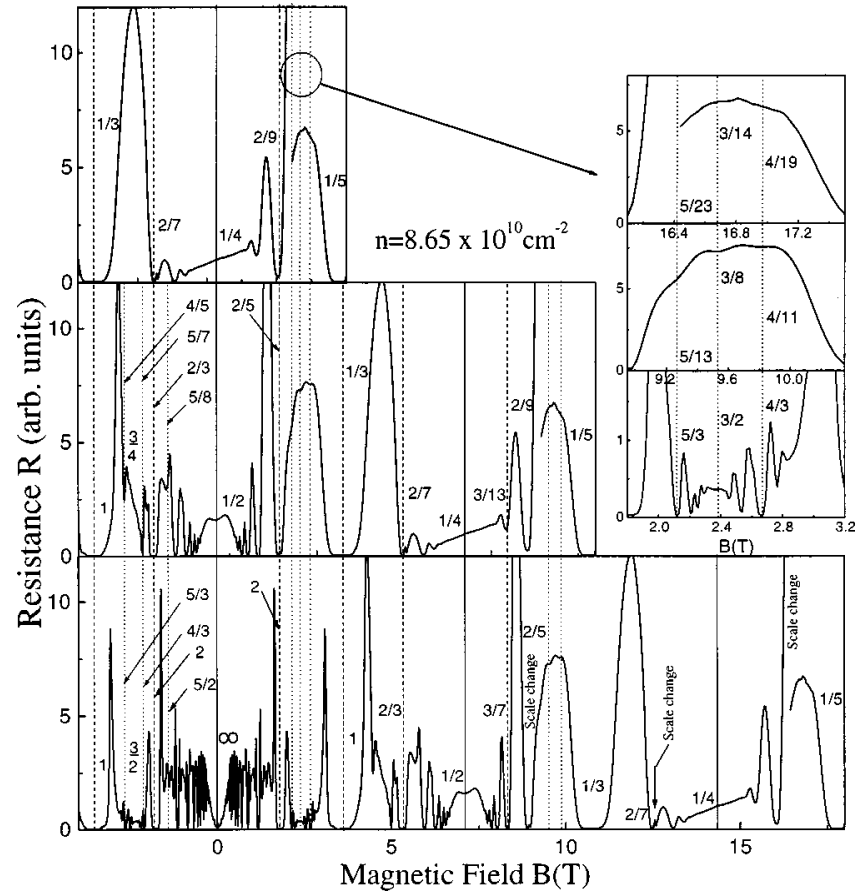

FIG. 19. Multiple sequences of FQHE states. Only magnetoresistance data are shown for clarity. In the middle and top panels, the bottom trace is shifted to the left by a magnetic field equivalent to the field at $\nu=1 / 2$ and $\nu=1 / 4$, respectively. The vertical lines show the self-similarity between different FQHE sequences. The right-hand inset is a blowup showing yet more developing FQHE states.

the starting point for multiple sequences of FQHE states. However, they themselves cannot be FQHE states. Yet the $5 / 2$ state is exactly that. It has all the characteristics of a FQHE state, including energy gap and quantized Hall resistance, in spite of its evendenominator classification (see Fig. 20). The $\nu=5 / 2$ state resides in a higher Landau level $(5 / 2=2+1 / 2)$, but this fact should not alter the simple reasoning. The Landau level below is energetically far removed and can be regarded as inert. Therefore the $5 / 2$ state is really a $1 / 2$ state in the next higher Landau level and should behave as such-but it does not. Discovered more than a decade ago, its true origin remains mysterious. With the advent of the CF model, the $\nu=5 / 2$ state has recently been revisited and a most tantalizing possibility has arisen.

Driven by many-particle interactions, the carriers at half filling of the next Landau level indeed bind two flux quanta each-just like their $\nu=1 / 2$ cousins. They form CFs and fill up the states, just as in Fig. 17. However, many-particle physics pushes those CFs further to a yet lower-energy ground state. In loose analogy to the formation of Cooper pairs in normal electron systems at low temperatures and their subsequent condensation into a superconducting state with an energy gap, these CFs form new $C F$ pairs which condense into a novel many-particle ground state. The resulting energy gap provides the essential ingredient for the observation of the characteristic FQHE features. This is a very exciting scenario, since it suggests that yet other, higher-order electron-electron correlations than those of the $\mathrm{CF} / \mathrm{CB}$ 


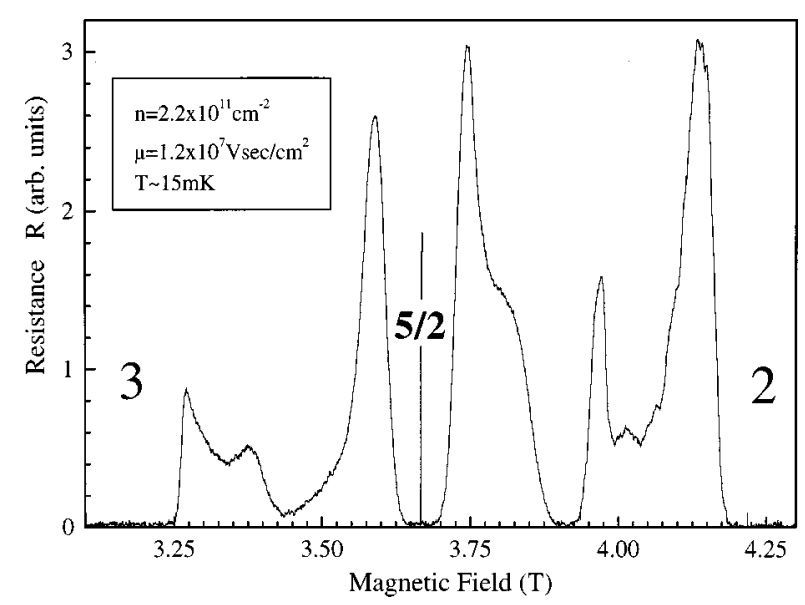

FIG. 20. FQHE at $\nu=5 / 2$. A FQHE state at such an even denominator fraction should not be allowed. The origin of the state remains unclear. An exciting possibility for the origin of this state is the formation of composite fermion pairs. These unpublished data were taken by J.-S. Xia and Wei Pan at the NHMFL in Gainesville, Florida.

model can play a decisive role. The properties of the resulting particles are also expected to be very unusual (non-Abelian).

At present it remains unclear whether the $5 / 2$ state is indeed of such an elaborate lineage, or whether some other, more mundane explanation will suffice. We will have to await future, more sophisticated experiments to tell us. If not the $5 / 2$ state, there is a good chance that some other, yet to be unearthed FQHE state may be of such an intricate origin. And there may well be states that we have not even imagined.

\section{CONCLUSIONS}

Two-dimensional electron systems in high magnetic fields reveal to us totally new many-particle physics. Confined to a plane and exposed to a magnetic field, such electrons display an enormously diverse spectrum of fascinating new properties: totally unexpected new electron states with fractional quantum numbers, the attachment of magnetic flux to electrons, new particles obeying either Bose or Fermi statistics, cancellation of exceedingly high magnetic fields, masses of purely electron-electron interaction origin, and possibly a strange, new process for particle pairing. These are but the most prominent of observations and implications. Most perplexing of all, such electrons create bizarre fractionally charged particles, without any individual electron splitting apart.

They are just electrons, although many of them. Indeed, "More is different!"

\section{EPILOGUE}

I am very honored having been chosen to share in this award of almost frightening proportions and I am grateful for receiving $1 / 3$ of this very special prize. Unfortunately, the yet more delightful $1 / 4$ version remained for- bidden. I attribute it all to an immense amount of good fortune throughout my life and the truly outstanding colleagues with whom it allowed me to work.

As so often, this award is being given to a lucky few, but it truly honors the immense progress that has been made in many-particle physics over the years-in particular in the physics of two-dimensional systems-and the large group of experimentalists and theorists who have brought it about. In this sense, I feel I share this award with so many of my colleagues and friends around the globe. To all of them I owe a great deal of gratitude.

As to our own contributions, the creators of materials remain the true heroes of the trade. Art Gossard and Willy Wiegmann fabricated the all-important sample in which the FQHE was discovered and many more after this event. Over the past decade or so, Loren Pfeiffer and Ken West brought the art and science of 2D material growth to new heights. It was in their samples that most of the exciting new discoveries in the FQHE were made. Kirk Baldwin's wizardry in the cleanroom and his screening of thousands of samples provided the underpinning to most of our experiments. Al Cho, John English, Jim Hwang, Mansour Shayegan, Charles Tu, Won Tsang, and Gunther Weiman also provided invaluable materials support.

I would not be here without the exceptional experimental skills and deep physical insights of postdocs, students, and collaborators at Bell Labs, Princeton University, and other institutions. They include Jim Allen, Ray Ashoori, Edwin Batke, Peter Berglund, Greg Boebinger, Albert Chang, Rui Du, Jim Eisenstein, Erich Gornik, Taisto Haavasoja, Rick Hall, Hong-Wen Jiang, Woowon Kang, Mikko Paalanen, Wei Pan, Aron Pinczuk, Zack Schlesinger, Joe Spector, Werner Wegscheider, Claude Weisbuch, Bob Willett, Jian-Sheng Xia, Andrew Yeh, and Amir Yacoby.

None of what has been discovered in experiments by many in the field I would have appreciated without my theorist friends and colleagues patiently teaching me FQHE physics. Particular insights I received from Nick d'Ambrumenil, Steve Girvin, Duncan Haldane, Bert Halperin, Song He, Jainendra Jain, Steve Kivelson, Bob Laughlin, Dung-Hai Lee, Peter Littlewood, Allan MacDonald, Rudolf Morf, Phil Platzman, Nick Read, Ed Rezayi, Ramamurti Shankar, and Steve Simon. There are many more, too numerous to list.

My new colleagues at Columbia University I thank for a warm reception in their midst. Dominique, my wife, I thank for her unceasing support and cheerfulness. I also thank my producer.

Finally, I want to thank my long-time collaborator and friend, Dan Tsui, in his characteristically few words: "Thanks for taking me to the dance."

\section{BIBLIOGRAPHY}

Several authoritative monographs have been written on the different topics addressed in this lecture, which point back to the extensive original literature. The following represents a sampling of such books. 
Physics in Silicon MOSFETs:

Ando, T., A. B. Fowler, and F. Stern, 1983, "Electronic properties of two-dimensional systems," Rev. Mod. Phys. 54, 437.

Molecular Beam Epitaxy:

Cho., A., 1994, Ed., Molecular Beam Epitaxy (AIP Press, Woodbury, New York).

Herman, M. A., and H. Sitter, 1998, Molecular Beam Epitaxy: Fundamentals and Current Status (Springer, Berlin).

Modulation Doping:

Schubert, E. F., 1993, Doping in III-V Semiconductors (Cambridge University Press, Cambridge, England).

\section{Hall Effect:}

Weiss, H., 1969, Structure and Application of Galvanomagnetic Devices (Pergamon, Oxford/New York).
Integral Quantum Hall Effect:

Chakraborty, T., and P. Pietilainen, 1995, The Quantum Hall Effects, Springer Series in Solid State Sciences No. 85 (Springer, New York).

MacDonald, A. H., 1990, Ed., Quantum Hall Effect: A Perspective (Kluwer Academic, Boston/Dordrecht).

Prange, R. E., and S. M. Girvin, 1990, Eds., The Quantum Hall Effect, 2nd edition (Springer, New York).

Fractional Quantum Hall Effect and Composite Fermions: Chakraborty, T., and P. Pietilainen, 1995, The Quantum Hall Effects, Springer Series in Solid State Sciences No. 85 (Springer, New York).

Das Sarma, S., and A. Pinczuk, 1997, Eds., Perspectives in Quantum Hall Effects (Wiley, New York).

Heinonen, O., 1998, Ed., Composite Fermions: A Unified View of the Quantum Hall Regime (World Scientific, Singapore). MacDonald, A. H., 1990, Ed., Quantum Hall Effect: A Perspective (Kluwer Academic, Boston/Dordrecht).

Prange, R. E., and S. M. Girvin, 1990, Eds., The Quantum Hall Effect, 2nd edition (Springer, New York). 